\title{
Scientific Realism meets Metaphysics of Quantum Mechanics*
}

\author{
Juha Saatsi
}

7th August 2017

\begin{abstract}
I examine the epistemological debate on scientific realism in the context of quantum physics, focusing on the empirical underdetermination of different formulations and interpretations of QM. I will argue that much of the interpretational, metaphysical work on QM transcends the kinds of realist commitments that are well-motivated in the light of the history of science. I sketch a way of demarcating empirically well-confirmed aspects of QM from speculative quantum metaphysics in a way that coheres with anti-realist evidence from the history of science. The minimal realist attitude sketched withholds realist commitment to what quantum state $|\Psi\rangle$ represents. I argue that such commitment is not required for fulfilling the ultimate realist motivation: accounting for the empirical success of quantum mechanics in a way that is in tune with a broader understanding of how theoretical science progresses and latches onto reality.
\end{abstract}

\section{Introduction}

The epistemological debate on scientific (anti-)realism has hitherto made little contact with philosophy of quantum physics. The latter mostly revolves around metaphysical controversies, recent developments of which raise a serious epistemic demarcation problem for the scientific realist: the realist needs to outline a principled way to distinguish empirically well-confirmed aspects of quantum physics from the quantum metaphysics that is a hotbed

\footnotetext{
${ }^{*}$ Presented at XII International Ontology Congress in San Sebastian. Forthcoming in Philosophers Think About Quantum Theory, Alberto Cordero (ed.), Springer.
} 
of controversy, disagreement, and (seemingly) radical speculation. Here I explore the nature of this demarcation problem and propose a way for the realist to approach it.

The scientific realism debate in general philosophy of science has a core epistemological dimension. According to realists we are justified in optimism regarding sciences' ability to represent the reality beyond observable phenomena. There is much variation in how optimistic we should be in this regard, but all realists are optimistic compared to anti-realists - empiricists and instrumentalists - who mainly place their trust in the sciences' deliverances regarding observable matters. The principal motivation for realism comes from the empirical success of science. What primarily drives the realists, in connection with theoretical sciences in particular, is the impressive predictive and explanatory success of our best theories. Arguably realism best accounts for this success, while antirealists' complete lack of optimism about theoretical progress and the reach of theory-based knowledge fits badly with the systematic success of science in yielding impressive predictions and seemingly good explanations.

Given the realists' emphasis on empirical success, one should expect this epistemological debate to rage in connection with quantum physics. For, on the one hand, quantum physics is one of the most successful areas of science of all time, and as such it elicits the realist intuition from empirical success as forcefully as anything in modern science: if there is anything we should want to be realists about, it is quantum physics. ${ }^{1}$ On the other hand, there are well-known challenges in pinning down what quantum physics purportedly says about the unobservable reality, making it exceptionally challenging to say what realism 'about the quantum' actually amounts to. In the light of this obvious tension, one would indeed expect quantum physics to be the battle ground for scientific realism. Digging into the large literature on scientific realism reveals very limited discussion focused on QM, however. ${ }^{2}$

While surprisingly little has been said about the implications of QM to the core epistemic issues in the realism debate, a huge deal has been written

\footnotetext{
${ }^{1}$ The realist intuition about $\mathrm{QM}$ is driven by countless novel predictions and explanatory achievements with respect to various distinct phenomena regarding atomic spectra, the periodic structure of elements, the band structure of the semiconductors, to name a few.

${ }^{2}$ There are some notable exceptions, of course, such as Cordero (2001), Cushing (1994), Barrett (2003), Belousek (2005), van Fraassen (1991).
} 
about the metaphysical implications of quantum physics, both in relation to quantum field theory and non-relativistic quantum mechanics. From the early days of quantum physics there has been extensive investigation into metaphysical issues that naturally arise from a realist outlook: what could the world literally described by quantum theories be like? Although this metaphysical question is naturally associated realism, it should not be identified with the essentially epistemological issues at the heart of the realism debate as they have been discussed in general philosophy of science. The latter issues - the topic of this paper-largely concerns the level of optimism we are justified in having towards quantum physics: In what sense (if any) are we justified in regarding QM as partially or approximately true, or (more generally) as latching onto unobservable reality? What kind of epistemic optimism about QM best coheres with the historicist anti-realist evidence regarding the pessimistic track-record of science in figuring out, through empirically highly successful theories, the fundamental nature of light, heat, gravity, and so on? What kind of epistemic optimism best coheres with the kind of underdetermination exhibited by the different variants of QM, or the likelihood of there being yet further variants hitherto unconceived by theorists?

The last of these questions has began to look particularly pressing of late, as metaphysical explorations of QM have taken an increasingly radical turn. A casual survey of the blooming metaphysical literature on QM raises pressing questions about the epistemological status of the competing claims regarding the nature of quantum state involved in various competing accounts. This is made all the more pressing by the striking lack of consensus amongst the experts: the current state of the art exhibits an unprecedented and radical underdetermination of the different world-views associated with a scientific theory that enjoys extraordinarily solid and varied empirical evidence. $^{3}$ Consequently many philosophers are alarmed by this underdetermination, because it appears to make it extraordinarily difficult to say what realism about $\mathrm{QM}$ amounts to.

A detailed examination of this interaction between the epistemology and the metaphysics of QM is long overdue. Here I will contribute to this task by delineating an epistemic attitude towards QM that coheres well not only

\footnotetext{
${ }^{3}$ This lack of consensus is equally true amongst philosophers of physics and physicists themselves. For one snapshot, see Schlosshauer et al. (2013).
} 
with the current state of affairs regarding quantum metaphysics, but also with (what I regard as) the most promising realist response to the anti-realist arguments from the history of science. I will outline a sense in which a realist can regard QM as more than a mere instrument for prediction, allowing for quantum theoretic understanding of various empirical phenomena. I will sketch a realist account of the empirical success of QM that demarcates empirically confirmed aspects of QM from quantum metaphysics, withholds commitment to what quantum state $|\Psi\rangle$ represents in the world, and avoids the brunt of the underdetermination problem.

\section{The epistemic demarcation problem}

Most scientific realists are naturally wary of the deeper reaches of metaphysics when it comes to delineating their epistemic commitments. While realists do not want to renounce metaphysics altogether in the way e.g. constructive empiricists do - think of van Fraassen (1980), for example - they generally acknowledge the pressure of the anti-realist arguments from either the history of science, or underdetermination, or both. Realists have toiled hard to render their epistemic commitments compatible with the features of past and present science emphasised by the anti-realists. In the light of these features it would be an obvious folly for the realist to commit to anything like the literal truth of any piece of current physics. More generally, realists should want to be less committal towards the more deeply metaphysical claims about the nature of reality, given the evidence of the unreliability of theoretical reasoning regarding such claims (e.g. Laudan 1981, Stanford 2006). For this reason prominent realist positions tend to radically reduce their epistemic commitments from a face-value reading of theoretical science, and only bank on what our theories say, for example, about the 'structure' of reality, or about the core causal features of the unobservable world (as opposed to peripheral metaphysical embellishments that scientists may appeal to for the sake of intelligibility). (I will examine these in further detail in Section 3.)

Recent philosophy of QM stands in stark contrast to this broadly antimetaphysical trend in the epistemology of scientific realism. Over the past couple of decades much of the philosophical work inspired by QM has gained an increasingly deep metaphysical flavour. Various radical ideas about the 
fundamental nature of reality have emerged as philosophers have attempted to spell out what the world described by the different variants of QM could be like if the theory is taken to truly represent the unobservable world behind the appearances. Many of these ideas are, prima facie, not only radical, but also rather indirectly connected to the actual scientific practice of using quantum theory to predict, manipulate, and explain things. For this reason I call them 'deeply' metaphysical.

Consider, for example, the debate about wavefunction realism. This debate about the nature of $|\Psi\rangle$ construed as a field-like feature of the worldas a literal reading of QM might suggest - was sparked by the recognition that the central posit of quantum mechanics, the wavefunction $|\Psi\rangle$, can be naturally interpreted as representing a field, but only if one takes seriously a very high-dimensional 'configuration' space, quite different from the familiar 3 -space (or $3+1$ spacetime) that we are directly acquainted with (Ney and Albert 2013). This line of thought immediately calls for a deep metaphysical account of quantum reality, since any interpretation of QM involving a realist commitment to $|\Psi\rangle$ thus construed cries out for a story of how the familiar 3space (whether as a real-but-not-fundamental space, or merely as a matter of appearances) 'emergences' from, or relates to, the very different space occupied by the wavefunction (see essays in Ney and Albert 2013).

Wavefunction realism is partly motivated by a fairly literal realist reading of the theory's formalism, but it is by no means forced upon the realist. A much discussed alternative is to demote the wavefunction to a different ontological category altogether, construing it rather as representing dynamicalcum-nomological features of a primitive ontology that occupies the familiar 3 -dimensional space. Relegating the ontological status of $|\Psi\rangle$ to a law-like feature of reality avoids the need to tell a deep metaphysical story of how what we see around us relates to (or emerges from) it, but instead it requires commitment to some kind of 'primitive stuff' (Maudlin 2007, Allori 2013). As to the nature of such 'stuff' occupying spacetime, a broad array of alternatives have been entertained by its advocates, ranging from relatively sparse momentary flashes in spacetime, to an esoteric mass density field, to individual particles that are entirely featureless in terms of their intrinsic properties, and so on. (For a brief review see e.g. Esfeld 2014.) There is a clear sense in which such primitive ontology is deeply metaphysical in that it is, indeed, posited as an ontological primitive, as opposed to being some- 
thing that needs to be written into the theory in the interest of improved predictions or explanations of empirical phenomena. Furthermore, regarding the wavefunction as a purely dynamical-cum-nomological feature of the world is itself an interpretational move that is far from obvious from the perspective of scientific practice. Such a move can be motivated in various ways philosophically, of course, but these largely hinge on deep metaphysical issues around laws of nature. ${ }^{4}$

There is no shortage of exciting alternatives in the quantum metaphysical marketplace: a further option is to regard the wavefunction merely as a representation of quantum superpositions, and to take the notion of quantum superposition itself at face-value, as a primitive and fundamental feature of reality. This is what the (in)famous Everettian many-worlds interpretation does. This line of thought is frequently defended by its advocates as being metaphysically light weight, introducing no further metaphysical posits or assumptions than what is to be found already in quantum physics (both in QFT and QM) pure and simple (Saunders et al. 2010, Wallace 2012, Vaidman 2014). To an extent this seems right: there is no need to posit a primitive ontology, or to adopt a particular stance regarding the metaphysics of laws of nature, for instance. On the other hand, the metaphysical picture of reality painted by the many-worlds interpretation relies on making sense of how effectively stable classical branches (or 'worlds') 'emerge' from the quantum multiverse. The Everettian understanding of 'classical worlds' as quasi-independent, stable patterns of an unimaginably richly structured fundamental quantum state of the universe relies not only on important features of quantum theory itself - environment-induced decoherence, in particular-but also on a deeply metaphysical account of how we can relate our (mostly 'classical') experiences to the quantum formalism that can describe the fundamental quantum multiverse.

This broad-brush run-through of the metaphysical aspects of the most central theoretical posit of QM, the wavefunction, in the most prominent realist interpretations of $\mathrm{QM}$ highlights a couple of things relevant to scientific realism. Firstly, as I will discuss further below, interpretations of QM, when spelt out in the level of detail required for their defence, become deeply metaphysical due to indispensably involving ideas about quantum

\footnotetext{
${ }^{4}$ See, e.g., Esfeld et al. (2015), Esfeld (2016), and Bhogal and Berry (2015), for discussions about Humean metaphysics as a backdrop to Bohmian QM.
} 
reality, and its relationship to observable features of the world, that are far removed from the actual scientific use of quantum theory to predict and explain empirical phenomena. Secondly, assuming that the different interpretations are underwritten by variants of QM that are all sufficiently empirically adequate, the realist faces a radical underdetermination of the metaphysical alternatives.

A natural knee-jerk realist response to the deeply metaphysical claims associated with QM is an incredulous stare. Should we seriously regard ourselves as having discovered, by carefully reflecting on an empirically extremely well confirmed scientific theory, that tables and chairs are stable, effectively non-interacting parts of an incredibly complex quantum multiverse? Or that they are in some sense reducible to a fundamental wavefunction that 'lives' in an extremely high-dimensional configuration space? Or that material objects are galaxies of relatively rare flashes associated with a sui generis dynamical collapse law that doesn't give rise to any new predictions? Such claims are all well and good as part of a metaphysical endeavour and as exploratory science, and for all we know one of them might depict the world more or less correctly. But in the light of the well-motivated antimetaphysical trend in the epistemology of scientific realism, a realist should be very wary of regarding any such claim as an empirically well-confirmed part of current science, falling under the realist's epistemic commitments. A realist operating with appropriate epistemic caution should rather regard such claims as belonging to some different, more speculative epistemic plane; claiming them to be part of scientific realist commitments is naturally greeted with an incredulous stare. ${ }^{5}$

Such incredulous stare is partly an indication of the high epistemic stakes of the radical revisions that interpretations of QM call for with respect to our everyday image of reality. We have, of course, become quite accustomed to the idea that the features of fundamental reality revealed to us by modern physics are unfathomably unlike our 'everyday reality'. But it is still reasonable to require that the evidence in support of any proposed metaphysical image of empirical reality should be commensurate with how revisionary that image is. The realists' worry about deeply metaphysical stories about quantum reality is that they are just that: just-so stories, devised so as to

\footnotetext{
${ }^{5}$ Note that none of this speaks against the rationality, meaningfulness, purposefulness, etc. of this kind of metaphysics. The point is purely epistemological.
} 
make sense of quantum mechanics literally construed, but without all the qualities that render scientific theories well confirmed by empirical evidence. This worry is bolstered by the fact that there is serious competition for any particular metaphysical image of quantum reality, making it harder to justify the adoption of any of the specific alternatives as being firmly a part of the scientific realist's commitments. ${ }^{6}$

Assuming the realist is rightly worried about any particular interpretation of QM, what epistemic attitude should she have towards it then? Should she give up realism about QM altogether in the light of the historical track record of theorists' unreliability in pinning down the metaphysics of empirically successful theories? This would, of course, give the realist game away entirely in connection with one of the empirically most successful areas of science, making it thereby also harder to maintain the realist motivation (which, recall, just turns on empirical success of science) in relation to other areas of science that deal with fundamental features of reality. Or can the realist appeal to some notion of 'approximate truth' or 'selective' realism that does not take QM at anything like its face value, but nevertheless maintains that the theory 'latches onto' reality in ways that account for its empirical success? There is significant pressure for the realist to find a way of doing this, but it is not easy, as it requires a principled criterion to demarcate justified epistemic commitments from what the realist should be inclined to view as 'metaphysical hubris', as far as the empirical evidence for the theory is concerned. Delineating such a criterion is thus the prime task for scientific realists in relation to QM.

In attesting to some such demarcation criterion scientific realists resist the kind of confirmational holism that naturalistic metaphysicians often appeal to. It has been argued - in the spirit of Quinean naturalistic 'indispensability argument', for example - that as realists we ought to commit to whatever theoretical assumptions indispensably contribute to our best scientific theories being the best: most predictive, explanatory, unifying, and simplest, etc. According to this line of thought, even if there is a distinction to be drawn between 'traditional' metaphysics and 'scientific metaphysics', when it comes to the latter it's a package deal (see Saatsi 2017 for a critical

\footnotetext{
${ }^{6}$ Peter Lewis (2016: 182) aptly summarises the state of play at the end of his book length review of quantum metaphysics: "Very little can be concluded unconditionally on the basis of quantum mechanics ... The best we can say is that not everything in our received classical worldview can be right."
} 
discussion). Even if relying on such confirmational holism is a way of doing metaphysics and justifying it as a rational endeavour, scientific realists should maintain that there is a more fine-grained demarcation to be done in relation to the epistemic reach of empirical evidence. The need for such a demarcation can be further motivated by considering, by way of an analogy, sensible realist attitudes towards metaphysics of other scientific theories, e.g. classical mechanics or biology. Various philosophers, in the spirit of naturalistic metaphysics, have drawn deeply metaphysical conclusions from classical mechanics, for instance. According to Quinean naturalists, scientific realists should say that numbers exist, given their indispensable theoretical and explanatory role in e.g. classical mechanics (e.g. Colyvan 2015; see Saatsi 2017 for criticism). According to Lewisian genuine modal realists, the modal features of classical physics can support very substantive theses in modal metaphysics (Lewis 1986; see also Williamson 2016). According to others, classical physics provides evidence for the reality of dispositions (e.g. Bigelow, Parketter 1990). According to the Humeans, the laws of classical physics are best-system regularities (e.g. Cohen and Callender 2009). Given that classical mechanics is a hugely successful theoretical framework empirically, a scientific realist attitude towards it is very well motivated. But does the empirical success of classical mechanics suggest that we should extend scientific realist commitments to the kinds of things that metaphysicians naturally associate with this theory's ontology: e.g. numbers, dispositions, particular metaphysics of laws? Friends of confirmational holism may think so (see e.g. Ellis 2009), but most philosophers engaged in the scientific realism debate rightly worry that there is a slide to speculative metaphysics here: notwithstanding their 'naturalistic', science-driven credentials, metaphysical claims about abstracta, the ontology of laws of nature, and modality, for example, transcend the empirical evidence in a way that outstrips the kind of empirical justification that realists rely on.

Resisting confirmational holism in this way requires more than a mere assertion, of course. I have said more to this effect elsewhere, e.g. with respect to mathematical platonism and scientific realism (Saatsi 2007, 2017). Here I just want to stress that many scientific realists do not want to slide into committing themselves to the various posits and explanations that the best metaphysical analyses may associate with that theory. In a similar vein, many scientific realists about biological theories of the evolution of proteins, 
say, or speciation processes, do not want to be saddled with having to pick a metaphysical account of species, or, proteins as natural kinds. This is largely due to the fact these metaphysical analyses are simply too indirectly connected to the empirical successes of the relevant theories that motivate realism in the first place. I think we should follow this intuition regarding quantum metaphysics as well: the realist should not feel pressed to choose between the competing metaphysical packages, because those metaphysical accounts are too indirectly connected to the empirical successes that motivate realism about QM in the first place. I will base my realist analysis on this intuition after critically reviewing, in the next section, some realist 'recipes' that one might try to appropriate to QM.

\section{Realist 'recipes' to the rescue?}

In response to the anti-realist challenges scientific realists have come up with various ways of demarcating the belief-worthy contents of science from what seems, in the light of the history of science in particular, rather more speculative and less trust-worthy. These demarcation principles are typically given in the abstract, recipe-like, so as to be applicable to different scientific theories, more or less independently of their specific subject matter or content. Familiar monikers include 'structural realism', 'entity realism', and 'semi-realism', each of which stands for a particular recipe for extracting from a given scientific theory its belief-worthy content, so as to allow the realist to be agnostic in a principled way about the rest of the theory, which can function as a mere heuristic crutch, or as a vehicle for a pleasing (but not necessarily truth-tracking) sense of intelligibility. One might think that the right way to approach the demarcation problem in the context of QM is also a matter of first identifying and then applying the right realist recipe.

I seriously doubt this is the best way for the realist to proceed, partly due to my general misgivings about the spirit of (what I have called) reciperealism (Saatsi 2015). Instead of aiming to provide an abstract recipe for extracting realist commitments from any given theory, it is better, I believe, to attend to the nature and subject matter of the theory in question, and ask how that theory's empirical successes are best accounted for in a realist spirit. There is no reason whatsoever to expect the answer to not vary from one theory (or area of science) to another in substantial ways that are 
not well captured by any abstract recipe (without such a recipe becoming rather contentless and disjunctive, at least). Rather, we should be open to the possibility that science itself, as well as realist commitments towards it, varies in such a way that the realist is better off by providing various more local exemplars of the sense in which the realist wants to commit herself to a given theory latching onto unobservable reality, without reducing that sense to any general characterisation of 'partial' or 'approximate' truth, or truth 'about structure' or 'detection properties' (Saatsi 2016). Let's now briefly consider some prominent realist recipes in relation to QM more specifically.

Structural ReAlism, as first proposed by John Worrall (1989) in connection with Fresnel's ether theory of light, relies on a distinction between a theory's structural content (or what it says about the structure of the world), on the one hand, and its non-structural content (or what it says about the nature of the world), on the other hand. Structural realism aims to capitalise on structural commonalities between different theories in order to provide a sense in which false theories - theories we struggle to view as 'approximately true' at the level of ontology - can nevertheless be taken to latch onto unobservable reality. Its advocates have suggested that structural commonalities between classical physics and quantum mechanics also fit this image, even if not as neatly as Worrall's main example does. ${ }^{7}$ Ladyman and Ross (2007, p. 94), for example, argue for this by offering some simple examples of "continuity in the mathematical structure of successive scientific theories", even across "the most radical cases of theory change in science, namely the transition from classical mechanics to Special Relativity, and the transition from classical mechanics to quantum mechanics."

The transition from classical mechanics to theories of relativity is a rich area of study, which has been discussed in the realism context in detail by Barrett (2008) and Saatsi (2016). The subtle correspondence between Newtonian gravity and Einstein's general theory of relativity is where the action is, given that the general theory is more fundamental than the special theory, and given the particularly stark ontological disparity between GTR and Newtonian gravity. Spelling out how the latter 'approximates' the structure of general theory of relativity arguably requires ideological resources specific to this area of physics, and properly accounting for the empirical success

\footnotetext{
${ }^{7} \mathrm{I}$ do not endorse the structuralist reading of the Fresnel-Maxwell theory-shift either (Saatsi 2005).
} 
of the classical theory, with its radically mistaken face-value ontology of gravitational forces acting at-a-distance, involves much beyond the notion that there is 'partial continuity of mathematical structure' between the two theories (see also Weatherall 2011). A realist's account of what makes Newtonian gravity empirically successful can ultimately have little in common with her account of what makes Fresnel's ether theory empirically successful. In particular, I (for one) do not see any useful abstract characterisation of structure that furnishes a unified explanatory sense in which Newtonian gravity and Fresnel's ether theories can both be regarded as 'getting the structure right'.

How about QM then? Here the structural realists point to various wellknown results that capture one or another aspect of the quantum-classical correspondence. For example, Ladyman and Ross (2007) mention Ehrenfest's theorems, and Bohr's 'correspondence principle' which requires that quantum mechanical models ought to mathematically reduce to their classical equivalents in the limit of large numbers of particles or when Planck's constant is taken to zero. ${ }^{8}$ French (2014) additionally points to the two theories' symmetry features, such as the relationship between Poisson brackets (classical) and Moyal brackets (quantum), which is naturally captured in group-theoretic terms. All these important relationships between the classical and the quantum - and there's plenty more, cf. Landsman (2007) — no doubt have a role to play in our best scientific understanding of the quantumclassical correspondence, as well as in a realist account of her epistemic commitments towards QM. But the account itself is again not reducible to the existence of such 'structural' correspondences. Rather, the account crucially involves sui generis dynamical features of QM, falling under the heading of decoherence, in particular, as I will discuss below (§4). Again, as we will see, the ideological resources required for a realist account of how classical physics relates to quantum physics are specific to quantum dynamics, and they involve much beyond the notion that there is a partial continuity of

\footnotetext{
${ }^{8}$ Ehrenfest's theorem shows how quantum mechanical expectation values of momentum and position operators obey an equation that structurally corresponds to Newton's equations of motion.
} 
mathematical structures between the two theories. ${ }^{9}$

Let's now move to the other side of the realist spectrum, as it were, where Hacking (1982, 1983) and Cartwright (1983), amongst others, have defended a very different kind of realist recipe for delineating realist commitments. The central idea of ENTITY REALISM is that realists should be committed to those (and only those) aspects of electrons, for example, that are required to account for scientists' ability to build finely-tuned 'electron spraying' instruments, such as the electron 'guns' that produce beams of polarised electrons, widely used in atomic and condensed matter physics. As Hacking's famous slogan has it, "if you can spray them, they are real." (1983: 23) Electrons are of course exactly the kind of thing that QM is used to study and understand, but Hacking regards such high-level quantum theoretical grasp of electrons entirely unnecessary for realism. Allegedly one simply need not appeal to a high-level theory to successfully build and operate an electron gun; all that is needed is knowledge of lower-level phenomenological causal regularities regarding electron behaviour.

There are well-known difficulties in spelling out what the entity realist is actually committed to in terms of our epistemic access to the unobservable entities that are 'sprayed' or manipulated to some empirical effect. Con-

\footnotetext{
${ }^{9}$ French (2014) furthermore takes the continuity and enrichment of the theories' symmetry features to signal the need to shift from (merely) epistemic structural realism (ESR) to ontological structural realism (OSR):

But if ESR is going to [incorporate the kinds of structures that matter in $\mathrm{QM}$, such as the structures encoding permutation symmetry], then it will have to take on the metaphysical consequences of this symmetry and those, I argue, lead us to abandon the notion of object, hidden or otherwise. In other words, if structural realism is to broaden its grasp and seize the kinds of structures that modern physics actually presents to us, then it is going to have to shift from ESR to OSR. (p.19)
}

As far as the scientific realism debate in general philosophy of science is concerned, this shift is in tension with the epistemological motivations that led to the idea of structura realism in the first place. The degree of epistemic humility that Worrall recommended by placing the realist's commitment to mere structure (as opposed to 'nature') is quite drastic from the point of view of 'standard' realism. If we take this degree of humility to be epistemically well motivated in the first place, and if we think that the distinction between structure and nature can be sensibly drawn, then we should see it as indicating scientists' unreliability in theorising about the nature of light and the nature of all other things (ultimately) quantum mechanical. But this level of scepticism about scientists' reliability to theorise about the fundamental nature of the world would also, it seems, speak against the philosopher's reliability to figure out whether the structural features of our best theories correspond to a structuralist ontology or otherwise. (See also Saatsi, 2009.) 
sider the entity realist's commitment to electrons, for instance. The idea is to capitalise on various kinds of instruments that use electrons effectively as a tool to some well-controlled effect. Spintronics provides a great example of modern instrumentation of this kind, relying on scientists' ability to manipulate electrons in intricate ways with electric and magnetic fields on the basis of their electric charge and a quintessentially quantum mechanical feature of spin. But what does the entity realist's existential commitment to spin- $\frac{1}{2}$ electrons amount to? An essential part of Hacking's realist brief is his advocacy of the causal theory of reference (as developed by Putnam 1975) to underwrite the truth of the existential claim 'electrons exist'. With the causal theory of reference the realist commitment to the referent of 'electron' does not presuppose descriptive accuracy of our current theory of electrons and spin: knowing of the existence of electrons can come apart from knowing (much) about what electrons are like.

Unsurprisingly, many commentators (e.g. Musgrave 1996) have found this difficult to stomach: what sense does the entity realist's existential claim make in the absence of corresponding commitment to our best theory of what these entities are actually like? In a broadly similar spirit, Stanford (2015) has argued that given how very thin the referential-cum-existential commitment is, antirealists can effectively agree that atoms and electrons probably exist, since all that really matters for the antirealists is whether or not we actually have some substantial knowledge of what electrons are like!

I think this line of criticism undermines reference-focused realism committed to the existence of entities called 'electrons'. However, as will become clear shortly, I prefer to think of realist commitment (at least in relation to fundamental physics, such as QM and spacetime theories) in a way that does not boil down to claims regarding existence. Entity realism is furthermore problematic, since it is not clear how the entity realist recipe accounts for the empirical success of QM at large. This difficulty is accentuated in the context of the metaphysics of QM. Faced with the radical divergence in the characterisation of spin, charge, and mass in Bohmian versus Everettian variants of QM, for example, the entity realist is all the more pressed to spell out the content of her commitment to electrons. For the Bohmian it is not the case that in spintronics electrons are manipulated on the basis of their intrinsic property spin, for instance. Rather, Bohmians can regard spin entirely as a feature of the quantum wavefunction (or whatever $|\Psi\rangle$ represents) - it is not 
a property instantiated by the particles, which only have positions (Brown et al. 1996, Norsen 2014). So, according to this variant of QM the 'entities' being manipulated in spintronics, for example, are not electrons, but $|\Psi\rangle$. By contrast, the Everettian regards spin as a property of the entities which instantiate it. In this way the metaphysical underdetermination leads to radical uncertainty as to what exactly is causally 'sprayed' or manipulated, and this effectively deflates the entity realist's epistemic commitment and her account of the empirical success of QM.

This is a challenge also for a more sophisticated, latter-day entity realism known as SEMIREALISM, which shifts the focus from entities to the core causal properties in an effort to say something more substantial about the objects of realist commitment. Semirealism, as developed by Chakravartty $(1998,2007)$ and $\operatorname{Egg}(2012,2015)$ in particular, is committed to knowledge of "causal properties that one has managed to detect" (Chakravartty 2007: 47). Semirealists contrast such 'detection' properties with 'auxiliary' properties, which are "any other putative properties attributed to particulars by theories" (ibid.), regarding which we are meant to be agnostic.

Detection properties are connected via causal processes to our instruments and other means of detection. One generally describes these processes in terms of mathematical equations that are or can be interpreted as describing the relations of properties. [One] can thus identify detection properties as those that are required to give a minimal interpretation of these sorts of equations. (ibid., p. 48, my emphasis.)

But what kind of 'minimal interpretation' in terms of causal detection properties can we give, for example, of the equations that predict the behaviour of a Stern-Gerlach detector, or quantum cyclotron, or a solid-state physics device in spintronics? On the face of it, it looks like our handle on spin risks being merely formal and mathematical if we just limit our analysis to the 'minimal' level of mathematical equations shared by the different variants and metaphysical interpretations of QM (cf. also Morrison 2007). Viewing spin as a 'detection property' of the entities involved already presupposes a layer of metaphysics unsupported by the empirical success at stake, and it is not even clear how well the causal ideology of semirealism fits the understanding of spin in e.g. Bohmian mechanics (Brown et al. 1996). 
One begins to worry that in order for the semirealist's epistemic commitments to be consistent with the varied landscape of quantum metaphysics, these commitments have to be so minimal that they do little to account for the empirical success of quantum physics. ${ }^{10}$

The prominent realist 'recipes' reviewed in this section have been developed largely independently of the specifics of quantum theory and the metaphysical issues that challenge realism about QM in particular. ${ }^{11} \mathrm{I}$ will next argue that instead of any of these popular recipes we should approach the epistemic demarcation problem by asking how to best account for the empirical success of QM given (i) its explanatory modal content (shared by the different variants of QM), and (ii) the emerging interpretation-independent understanding of the relationship between QM and classical physics.

\section{Accounting for the empirical success of QM}

Many have thought the underdetermination problem challenges realism about QM, because the realist needs to pick a specific (realist) interpretation of QM in order to express her epistemic commitments regarding the relationship between QM and reality, but she has no empirical grounds for doing so. ${ }^{12}$ The challenge can appear rather pressing, for how could one claim to hold a realist attitude towards a theory without being able to say what the world is like according to it? Antirealism beckons, unless one can respond to the epistemic demarcation problem in a way that does not require choosing any specific interpretation.

I think this way of presenting the realist predicament is much too stark. I will now argue that the realist can hope to provide a satisfactory response to the epistemic demarcation problem while maintaining a kind of quietism about the interpretational issue. The resulting position is somewhat minimal

\footnotetext{
${ }^{10}$ Saatsi (2012) notes similar worries about semi-realist's ability to provide a substantial account of the empirical successes of ether theories.

${ }^{11}$ Ontic structural realism is a clear exception to this, but for the reasons given (cf. footnote 9) I am concerned with structural realism merely as a form a epistemic humility.

${ }^{12}$ Musgrave (1992) argues that a realist can appeal to general metaphysical criteria to eliminate all but one competing interpretation. In the light of the anti-metaphysical trend in the contemporary realism debate such general metaphysical criteria for theory-choice are difficult to motivate as a reliable source of justification, however.
} 
in its epistemic commitments, yet sufficiently realist in its spirit. Also, it is no more minimal than the epistemic commitments that would have been appropriate for the Newtonians or the ether theorists, for example, regarding their empirically successful theories (Saatsi 2015, 2016). The key is to identify when theorising about quantum phenomena slides so deep into metaphysics that goes beyond empirically justified realist commitments. Although it is difficult to draw sharp line here, we can reflect on the general principles that determine the answer. I view the following, in particular, as hallmarks of deeply metaphysical aspects of scientific theories: (A) the inability to give rise to new predictions, and (B) the inability to support bona fide scientific explanations.

The theoretical framework of QM is hugely successful, of course, in terms of both its predictive capacity and its explanatory power with respect to various phenomena, and at minimum the realist is committed to claiming that these varied empirical successes are due to QM latching onto unobservable reality. But the realist can regard the extant attempts to spell out what $|\Psi\rangle$ represents as deeply metaphysical - thus lying outside her epistemic commitments - because they neither generate new testable predictions nor support explanations that are bona fide scientific. Therefore, as they stand, the realist can deem the different interpretations of $|\Psi\rangle$ as an exercise in metaphysics or exploratory science that transcends her epistemic commitments.

The realist's epistemic commitments are thus determined by what she thinks accounts for the theory's undeniable empirical successes. Presumably the realist is unable to provide a fully fledged account of these successes in the absence of a complete grasp of the relationship between quantum and classical physics, which would involve both a complete quantum theoretical understanding of this inter-theoretic relationship and the role of decoherence therein, as well as the lack of clear empirical evidence for certain interpretational aspects of the quantum-classical correspondence. (A complete account of the theory's empirical success would of course involve also the correct metaphysics of $|\Psi\rangle$.) But this does not mean that the realist cannot say anything about what accounts for QM's empirical success, since she can point to: (i) the emerging, interpretation-independent, scientific understanding of the relationship between the quantum features of reality and the classical features; and (ii) those modal features of scientific explanations 
that are supported by QM independently of the specific interpretations of $|\Psi\rangle$.

Let me now elaborate on this sketch, beginning with (A): the inability of deep metaphysics to give rise to new predictions. We can begin with the truism that the work on quantum metaphysics and the measurement problem by and large does not generate any new predictions. The aim of this work is rather to make sense of QM and to spell out what the world could be like according to this or that empirically adequate variant of the theory. This is all well and good as a foundational and metaphysical endeavour, but there is an obvious sense in which interpretational and foundational work is not responsible for QM's immense predictive and practical successes that motivate realism in the first place. ${ }^{13}$ To the extent that the empirical successes of quantum physics can be regarded as independent from such metaphysical-cum-foundational work, the realist is justified in bracketing the fruits of that labour (as they stand) outside of her epistemic commitments.

There is a long tradition in the realism debate at large, as well as in the philosophy of QM more specifically, to think otherwise. This has been partly motivated by shortage of coherent realist interpretations of QM and lack of understanding of the quantum-classical correspondence, and partly by presuppositions about what realism about the quantum should amount to. It has been commonly presupposed that realists should be able to tell us what the nature of reality (quantum or otherwise) is like; that they should be able to specify what the key theoretical terms (e.g. $|\Psi\rangle$, 'entanglement', etc.) refer to; that they should be able to tell what the world must be like to underwrite the theory's approximate truth. However, more recent developments on the epistemic side of the realism debate have driven realists - myself, at leastto forgo these kinds of commitments in reaction to the challenges from the history of science and elsewhere.

In the light of the history of science it is simply indefensible, I think, to maintain the traditional realist hope that our current best theories reveal us the nature of reality in this way. One should delineate one's realist commitments towards current science in a way that is applicable to, for example, Newtonian gravity in the day of Newton, and to Fresnel's theory of light in his day, in advance of the subsequent scientific developments that we can

\footnotetext{
${ }^{13}$ This is of course not to say that such work cannot become responsible for such successes, but this potential has no bearing on our current epistemic commitments.
} 
now (with the benefit of hindsight) employ to account for those past theories' empirical successes from our current vantage point. (See Stanford 2006, 2015; Saatsi 2015.) Such historical applicability of the realist perspective is forced upon us, lest one is to argue for some kind of exceptionalism about the epistemic standing of current fundamental physics. ${ }^{14}$ If one is inclined to jettison the traditional realist ambition in favour of a more minimal realist attitude that is compatible with the historicist anti-realist arguments, then one can also give up on the idea that realist commitments towards QM should automatically include a stance towards metaphysical matters of the sort required to 'make sense of QM' and to say what the world is fundamentally like according to it.

Let's now move on to consider (B): the second hallmark of deep metaphysicsits inability to support bona fide scientific explanations. Drawing a distinction between scientific and metaphysical explanations is a subtle business, but the core idea here is quite simple: scientific explanations turn on counterfactual information that by scientific lights is regarded as justified by empirical evidence. By contrast, the different interpretations of QM furnish metaphysical explanations in terms of the nature of $|\Psi\rangle$ and its relationship to observable matters, such that the explanatory information in question does not boil down to counterfactual information that is empirically justified by the lights of science. Drawing the distinction in these terms is motivated by recent accounts of scientific explanation, which explicitly capitalise on counterfactual information of this sort: arguably many scientific explanations, causal and non-causal alike, involve counterfactual information that links the values of an explanans variable to the state of the explanandum so as to answer change-relating what-if-things-had-been-different questions (e.g. Woodward 2003, French and Saatsi forthcoming, Jansson and Saatsi forthcoming). Arguably the different metaphysical accounts of $|\Psi\rangle$ do not provide further explanatory information of this sort, since they do not involve further explanans variables, such that some empirical explanandum could be regarded as depending on those variables in an empirically wellgrounded way. ${ }^{15}$

\footnotetext{
${ }^{14}$ The ideas behind (epistemic) structural realism have been an important step in this direction, but as already indicated in Section 3, I don't think it's the best way of spelling out realist commitments in general.

${ }^{15}$ Neither are they based on mathematical or logical necessities of the sort that according to Lange (2013) can support explanations of empirical phenomena without explanatory
} 
Metaphysical explanations supported by interpretations of QM can be distinguished from scientific explanations in epistemological terms, even if they have the same basic structure as scientific explanations. For example, Schaffer (forthcoming) argues that metaphysical and scientific explanations share the same tripartite structure of 'source', 'principle', and 'result', where the connecting explanatory principle can be e.g. causation (in science) and grounding (in metaphysics), and the explanatory connection can be represented by structural equation models that capture how variation in the source is explanatorily connected to variation in the result. ${ }^{16}$ Applying this unifying analysis to QM, Schaffer argues that it allows us to make sense of the wavefunction realists' metaphysical explanation of how objects and facts about 3-space are grounded in the fundamental wavefunction. (This is Schaffer's response to the worry that Maudlin (2010) amongst others have voiced about the impossibility of comprehending how the fundamental wavefunction ontology can give rise to regularities in 3-space.) My present point is that even if we can make sense of the nature of the quantum metaphysical explanation in these broadly modal terms, the explanation need not be regarded as involving the kind of explanatory connection for which we have good empirical evidence, and hence the realist should still deem it deeply metaphysical.

Which explanatory successes of QM should the realist aim to account for? I think the answer to this question is determined by scientists' own assessment of the various explanations that QM furnishes: the realist can take the scientific community as a (hopefully) reliable judge as to which quantum mechanical explanations should be regarded as undeniable successes. Healey (2015: 2) rightly observes that "the continuing failure to agree on any specific realist interpretation or reformulation [of QM] contrasts strikingly with the widespread acceptance in the scientific community of the enormous explanatory power of contemporary quantum theory". Healey then goes on to

counterfactuals.

${ }^{16}$ As Schaffer (forthcoming, p. 2) explains:

With causal explanation, there is the structure of cause (such as the rock striking the window), law (laws of nature), and effect (such as the shattering of the window). Metaphysical explanation has a parallel structure, involving ground (the more fundamental source), principle (metaphysical principles of grounding), and grounded (the less fundamental result). One finds a similar structure with logical explanation, involving premise, inference rule, and conclusion. 
discuss in detail accepted quantum theoretic explanations of single-particle interference phenomena, the stability of matter, and interference of BoseEinstein condensates. It is natural for the realist to rely on scientists' own assessment of these kinds of explanatory successes, since in the present dialectic it is the explanatory success of science (as opposed to metaphysics of science) that the realist aims to account for, and realists typically furthermore argue that scientists' own assessments of explanations are a reliable guide to theoretical progress.

It is notable that in providing quantum theoretic explanations of various phenomena scientists by and large do not feel the need to appeal to any particular explication of the nature of the quantum state. Also, more specific explanations of e.g. interference phenomena that indispensably turn on specific interpretational choices can be ruled out: they do not enjoy sufficiently wide-spread scientific agreement, since there is no solid empirical evidence for preferring one such explanation over the alternatives. I furthermore conjecture that the explanations that physicists largely agree upon are associated with reasonably precise and empirically well-founded counterfactual information, amenable to a counterfactual account of explanation and explanatory understanding (cf. Healey 2015). The realist can thus account for these explanatory successes in terms of QM getting the appropriate explanatory counterfactuals right, since this is what really matters for providing the explanatory information, and this can be achieved even when the theory we are operating with is only in some sense a limited 'approximation' to a better theory we don't yet have (and may never have). Whatever the theory says about the world beyond those counterfactuals is supererogatory with respect to accounting for its explanatory success. In a similar way a minimal realist can capture the explanatory successes of Newtonian mechanics and gravity, for example. From a perspective that takes GTR seriously, the posit of gravitational force, acting at a distance, or Newtonian absolute simultaneity, are not involved in accounting for the explanatory successes of Newtonian gravity. By the same token, these genuine explanatory successes are not undermined by the fact that there are various features of the world that the theory simply got wrong (Woodward 2003b, Bokulich 2016). It is in this same spirit that the realist can regard the metaphysical accounts of the quantum state as simply supererogatory in accounting for the explanatory successes of QM. 
To summarise, the appropriate realist response to the underdetermination challenge is to insist that the underdetermination takes place at the level of deep metaphysics going beyond realist commitments. The different variants of QM, in as far as they are empirically adequate, all latch onto reality in ways that account for their empirical success. Getting a more complete handle on this account is something that will gradually take place alongside future scientific advancements, and the right realist attitude towards QM is a matter of confidence and optimism in there being such an account to be worked out. The underdetermination problem is thus neutralised by a natural, substantial reduction in realist commitments, which is furthermore incentivised (for reasons given in Section 2) independently of the underdetermination challenge: even without the predicament of underdetermination the issue of separating the empirical wheat from the metaphysical chaff looms large (cf. Saatsi 2016). ${ }^{17}$

\section{$5 \quad$ Is this realism at all?}

One may feel that the epistemological stance sketched above is insufficiently realist. At least a couple of potential objections immediately crop up. First, what can we be realists about, if we bracket the different interpretations of $|\Psi\rangle$ as 'deep metaphysics' that transcend realist commitments? Secondly, what about the notorious measurement problem: how do we respond to it if we cannot help ourselves to the resources afforded by a fully fledged interpretation? Isn't solving the measurement problem a sine qua non for realism about QM?

Let's address the latter question first. There is, of course, a long standing tradition to think that a realist must give an account of what the quantum state represents in the world in order to deal with the measurement problem. This line of thought goes as follows. The standard ('textbook') QM, which incorporates the collapse postulate, is not amenable to a realist attitude

\footnotetext{
${ }^{17}$ For the sake of the argument and to illustrate, consider a possible counterfactual history where theorists only ever come up with the de Broglie-Bohm variant of QM and are unable to conceive of any serious alternatives to it. In the light of the anti-realist challenges from the history of science, the realist would face the epistemic demarcation problem even in the absence of any actual alternative underdetermined by the empirical evidence. In particular, the realist should not want to commit herself to an interpretation of $|\Psi\rangle$ as a peculiar law of nature, say, even if the de Broglie-Bohm variant of QM seemed like the only game in town.
} 
towards the dynamics of the theory, given the irreducible role played by the notion of measurement as yielding determinate observable measurement outcomes. The upshot, then, is that the orthodox QM, unvarnished with a 'realist interpretation', is best regarded as a mere instrument or recipe for making predictions. Avoiding such blatant anti-realism about QMas the realist desires - thus requires articulating and defending a variant of QM that does not involve the problematic collapse postulate inconsistent with the unitary quantum dynamics. That is, it requires articulating and defending a variant of QM amenable to a realist interpretation.

This standard story is too black-and-white, however, from the perspective of the kinds of fairly minimal and unambitious epistemological stances that many (e.g. structural) realists have adopted towards physics in general. Doing without the collapse postulate and defending a particular realist interpretation of $\mathrm{QM}$ are not one and the same thing. There are degrees of epistemological commitment that fall between adhering to the 'orthodox' QM with the collapse postulate, on the one hand, and committing to one or another variant/interpretation that does without it, on the other. Since the collapse postulate drops out of the picture in all (current) variants of QM seriously entertained by the realists, and since it arguably plays less of a role in the physicists' actual (more interpretation-independent) use of QM than the above line of thought suggests, it is natural to consider what can be said of the relationship between classical and quantum physics independently of any 'realist interpretation' ${ }^{18}$

It is particularly noteworthy that the unitary quantum dynamics by itself gives rise to environment-induced decoherence that is at the heart of many physicists' own understanding of the relationship between quantum and classical physics, in a way that is independent of any particular variant of QM (Schlosshauer 2007, Wallace 2012). Decoherence does not 'solve' the measurement problem in and of itself, of course, because it does not answer the metaphysical question of what $|\Psi\rangle$ represents. An answer to the metaphysical question is a 'necessary coda' (as Rosaler 2016 puts it) to any decoherence-based account of how (approximately) classical dynamical and kinematical structures are compatible with a fundamentally quantum real-

\footnotetext{
${ }^{18}$ Wallace (forthcoming) convincingly argues against philosophers' commonplace idea that collapse (or "projection') postulate is central to "orthodox" or "standard" QM that physicists employ in practical applications.
} 
ity. But, as Rosaler (2016) forcefully argues, one can say a good deal about classical-quantum correspondence even without the interpretational coda. More specifically, Rosaler argues for the potential for combining technical, foundational understanding of (i) decoherence, (ii) Ehrenfest's Theorem for open quantum systems, and (iii) a decoherence-compatible mechanism for collapse, in providing a local interpretation-neutral reduction between particular models of quantum and classical theories. Such a foundational programme points to the kind of interpretation-independent account of the empirical success of quantum mechanics that a realist like myself is committed to being there to be fully worked out as a part of future science. Although metaphysical issues concerning effective 'wave function collapse' and the ontology underpinning a scientifically kosher reductionist account is a necessary part of a fully fledged account of quantum-classical correspondence, Rosaler shows how these concerns can be effectively decoupled from "the bulk of technical analysis necessary to recover localised, approximately Newtonian trajectories from quantum theory" (p. 54). Correspondingly, in defending a realist attitude towards QM one does not need to solve the measurement problem - to provide the interpretational coda - since the interpretation-neutral part of the analysis is enough to support the realist belief that the theory's empirical success are due to, and can be accounted for, in terms of it latching onto the unobservable reality in appropriate ways. As Rosaler (2016: 59) puts it:

$[\mathrm{O}]$ ne can go quite far in providing a quantum-mechanical account of classical behavior without taking on the speculative commitments associated with some particular interpretation of quantum theory. Of course, we must also keep in mind that at most one of these interpretation-specific accounts can be correct as a description of the collapse mechanism that nature itself employs.

What does it take to account for a theory's empirical success exactly? This is an important question that requires further analysis. I will limit myself here to noting a couple of complicating issues. For one, the realist's optimism about a theory should be compatible with the possibility that we can more fully account for its empirical success only with the benefit of hindsight furnished by a currently unavailable successor theory that advances on 
our present science. (Else, the history of science contains powerful cases against realism.) But even in the absence of such future science the realist can commit to optimism about there existing such an account, and one that we can hopefully give in due course. That is, the realist can express confidence in the fact the theory relates to reality in objective ways that are responsible to its success. This kind of attitude towards QM is clearly different from instrumentalism or empiricism; hence I associate it with the realist tradition. Secondly, there is a difference between a realist account of empirical success, which can be given in scientifically kosher terms that do not transcend the reach of available empirical evidence, and a complete account of empirical success, which can only be given from a (scientifically chimerical) omniscient point of view, involving also deep metaphysics of reality.

One may be inclined to associate more lofty ambitions with 'scientific realism', of course. For example, one may think that a scientific realist attitude towards a theory must entail knowledge claims about what kinds of things are real; what there is; what our theoretical terms refer to (see e.g. Stanford 2015). Admittedly, by those lights the optimistic epistemic stance I have sketched does not qualify as realism, given that this stance indeed does not defend realism about the quantum wavefunction, or spin, or quantum particles, in anything like the way that standard 'convergent realism' does regarding a theory's central posits (cf. Laudan 1981). If one is strongly inclined to stipulate that 'scientific realism' must entail such commitment, a new label is needed for the kind of optimism that I have argued for. ('Theory-progressivism' perhaps?) As I see it, this optimism should be directed towards a more abstract sense in which we are justified in regarding QM as latching onto unobservable reality in ways that drive the theory's empirical success, both predictive and explanatory. This latching is a matter of the theory's central kinematic and dynamical aspects representing the world's kinematical and dynamical structures sufficiently faithfully, in appropriate respects, along the lines studied, e.g., by Rosaler (2016) and Landsman (2007), and getting right the appropriate modal features of the world that underwrite undeniable scientific explanations of quantum phenomena. 
Acknowledgments. Support from Arts and Humanities Research Council (as part of Scientific Realism and the Quantum project) is gratefully acknowledge. I received helpful feedback on presentations in Leeds, San Sebastian, NYU Abu Dhabi. Special thanks to Fabio Ceravolo, Kevin Coffey, Steven French, Simon Newey, Kohei Morita, and Peter Vickers for helpful discussions and feedback an earlier drafts.

\section{References}

Allori, V. Primitive ontology and the classical world. In R. Kaster (Ed.), Quantum Structural Studies: Classical Emergence from the Quantum Level.

Allori, V. (2013). Primitive ontology and the structure of fundamental physical theories. In D. Z. Albert and A. Ney (Eds.), The Wave Function: Essays in the Metaphysics of Quantum Mechanics, pp. 58-75. Oxford: Oxford University Press.

Barrett, J. A. (2003). Are our best physical theories (probably and/or approximately) true. Philosophy of Science 70(5), 1206-1218.

Barrett, J. A. (2008). Approximate truth and descriptive nesting. Erkenntnis $68(2), 213-224$.

Belousek, D. W. (2005). Underdetermination, realism, and theory appraisal: An epistemological reflection on quantum mechanics. Foundations of Physics 35(4), 669-695.

Bhogal, H. and Z. Perry (2015). What the humean should say about entanglement. Nô̂s, 1-21.

Bigelow, J. and R. Pargetter (1990). Science and Necessity. Cambridge: Cambridge University Press.

Bokulich, A. (2016). Fiction as a vehicle for truth: Moving beyond the ontic conception. The Monist 99(3), 260-279. 
Brown, H., A. Elby, and R. Weingard (1996). Cause and effect in the pilotwave interpretation of quantum mechanics. In J. T. Cushing and e. al. (Eds.), Bohmian mechanics and quantum theory: An appraisal, pp. 309319. Dordrecht: Kluwer.

Cartwright, N. (1983). How the laws of physics lie. Oxford: Oxford University Press.

Chakravartty, A. (1998). Semirealism. Studies in History and Philosophy of Science Part A 29, 391-408.

Chakravartty, A. (2007). A Metaphysics for Scientific Realism: Knowing the Unobservable. Cambridge: Cambridge University Press.

Cohen, J. and C. Callender (2009). A better best system account of lawhood. Philosophical Studies 145(1), 1-34.

Colyvan, M. (2015). Indispensability arguments in the philosophy of mathematics. Stanford Encyclopedia of Philosophy.

Cordero, A. (2001). Realism and underdetermination: some clues from the practices-up. Philosophy of Science 68, S301-S312.

Cushing, J. T. (1994). Quantum mechanics: historical contingency and the Copenhagen hegemony. Chicago: University of Chicago Press.

Cushing, J. T. (1996). Quantum mechanics and underdetermination. In I. Douven and L. Horsten (Eds.), Realism in the Sciences: Proceedings of the Ernan McMullin Symposium, Leuven 1995. Leuven University Press.

Egg, M. (2012). Causal warrant for realism about particle physics. Journal of General Philosophy of Science 43(2), 259-280.

Egg, M. (2016). Expanding our grasp: Causal knowledge and the problem of unconceived alternatives. British Journal for the Philosophy of Science 67(1), 115-141.

Ellis, B. (2009). Metaphysics of Scientific Realism. Acumen.

Esfeld, M. (2014). The primitive ontology of quantum physics: Guidelines for an assessment of the proposals. Studies in History and Philosophy of Modern Physics 47, 99-106. 
Esfeld, M., D. Lazarovici, V. Lam, and M. Hubert (2015). The physics and metaphysics of primitive stuff. The British Journal for the Philosophy of Science, axv026.

French, S. (2014). The structure of the world: Metaphysics and representation. Oxford: Oxford University Press.

French, S. and J. Saatsi (2017). Symmetries and explanatory dependencies in physics. In A. Reutlinger and J. Saatsi (Eds.), Explanation Beyond Causation: Philosophical Perspectives on Non-Causal Explanations. Oxford: Oxford University Press.

Frigg, R. and I. Votsis (2011). Everything you always wanted to know about structural realism but were afraid to ask. European Journal of Philosophy of Science 1(2), 227-276.

Hacking, I. (1982). Experimentation and scientific realism. Philosophical Topics 13, 71-87.

Hacking, I. (1983). Representing and Intervening: Introductory Topics in the Philosophy of Natural Science. Cambridge: Cambridge University Press.

Healey, R. (2015). How quantum theory helps us explain. The British Journal for the Philosophy of Science 66, 1-43.

Jansson, L. and J. Saatsi (Forthcoming). Explanatory abstractions. British Journal for the Philosophy of Science.

Ladyman, J. and D. Ross (2007). Everything Must Go: Metaphysics Naturalised. Oxfords: Oxford University Press.

Landsman, N. P. (2007). Between classical and quantum. In J. Butterfield and J. Earman (Eds.), Handbook of the Philosophy of Science: Philosophy of Physics, pp. 417-554. Amsterdam: Elsevier.

Lange, M. (2013). What makes a scientific explanation distinctively mathematical? British Journal for the Philosophy of Science 64, 485-511.

Lewis, P. (2016). Quantum Ontology: A Guide to the Metaphysics of Quantum Mechanics. Oxford: Oxford University Press. 
Maudlin, T. (2010). Can the world be only wavefunction? In S. Saunders, J. Barrett, A. Kent, and D. Wallace (Eds.), Many Worlds? Everett, Quantum Theory, 8 Reality, pp. 121-143. Oxford: Oxford University Press.

Maudlin, T. W. (2007). Completeness, supervenience and ontology. Journal of Physics A: Mathematical and Theoretical 40(12), 3151.

Morrison, M. (2007). Spin: All is not what it seems. Studies in History and Philosophy of Modern Physics 38(3), 529-557.

Musgrave, A. (1992). Realism about what. Philosophy of Science 59(4), 691-697.

Musgrave, A. (1996). Realism, truth and objectivity. In R. Cohen, R. Hilpinen, and Q. Renzong (Eds.), Realism and Anti-Realism in the Philosophy of Science, pp. 19-44. Dordrecht: Kluwer.

Ney, A. and D. Z. Albert (2013). The wave function: Essays on the metaphysics of quantum mechanics. Oxford: Oxford University Press.

Norsen, T. (2014). The pilot-wave perspective on spin. American Journal of Physics 82, 337-348.

Putnam, H. (1975). Philosophical Papers: Volume 2, Mind, Language and Reality, Volume 2. Cambridge: Cambridge University Press.

Rosaler, J. (2016). Interpretation neutrality in the classical domain of quantum theory. Studies in History and Philosophy of Modern Physics 53, $54-72$.

Ruetsche, L. (Forthcoming). Getting real about quantum mechanics. In J. Saatsi (Ed.), Routledge Handbook of Scientific Realism. Routledge.

Saatsi, J. (2007). Living in harmony: Nominalism and the explanationist argument for realism. International Studies in the Philosophy of Science 21 (1), 19-33.

Saatsi, J. (2012). Scientific realism and historical evidence: Shortcomings of the current state of debate. pp. 329-340. Springer.

Saatsi, J. (2015a). Historical inductions, old and new. Synthese, 1-15. 
Saatsi, J. (2015b). Replacing recipe realism. Synthese, 1-12.

Saatsi, J. (2016). What is theoretical progress of science. Synthese, 1-21.

Saunders, S., J. Barrett, A. Kent, and D. Wallace (Eds.) (2010). Many Worlds? Everett, Quantum Theory, $\&$ Reality. Oxford: Oxford University Press.

Schaffer, J. (Forthcoming). The ground between the gaps. Philosophers' Imprint.

Schlosshauer, M. (2007). Decoherence and the Quantum-To-Classical Transition. Springer.

Schlosshauer, M., J. Kofler, and A. Zeilinger (2013). A snapshot of foundational attitudes toward quantum mechanics. Studies in History and Philosophy of Modern Physics 44(3), 222-230.

Stanford, P. K. (2006). Exceeding Our Grasp: Science, History, and the Problem of Unconceived Alternatives. Oxford: Oxford University Press.

Stanford, P. K. (2015). "atoms exist" is probably true, and other facts that should not comfort scientific realists. Journal of Philosophy 112(8).

Vaidman, L. (2014). Many-worlds interpretation of quantum mechanics. Stanford Encyclopedia of Philosophy.

van Fraassen, B. C. (1980). The Scientific Image. New York: Oxford University Press.

van Fraassen, B. C. (1991). Quantum Mechanics: An Empiricist View. Oxford: Oxford University Press.

Wallace, D. (2012a). Decoherence and its role in the modern measurement problem. Philosophical Transactions of the Royal Society A 370(1975), 4576-4593.

Wallace, D. (2012b). The Emergent Multiverse: Quantum Theory according to the Everett Interpretation. Oxford: Oxford University Press.

Wallace, D. (Forthcoming). What is orthodox quantum mechanics? 
Weatherall, J. (2011). On (some) explanations in physics. Philosophy of Science 78(3), 421-447.

Williamson, T. (2016). Modal science. Canadian Journal of Philosophy 46.

Woodward, J. (2003a). Experimentation, causal inference, and instrumental realism. In H. Radder (Ed.), The Philosophy of Scientific Experimentation, pp. 87-118. Pittsburgh: University of Pittsburgh Press.

Woodward, J. (2003b). Making things happen: A causal theory of explanation. Oxford: Oxford University Press.

Worrall, J. (1989). Structural realism: the best of both worlds? Dialectica $43,99-124$. 